\title{
Patella dislocation with unusual osteochondral fracture, whilst playing virtual reality game
}

\begin{abstract}
Case: We present case of a 37-year old male who sustained Patella dislocation with an unusual osteochondral fracture (OCF) involving the posterolateral femoral condyle, whilst playing a virtual game, wearing virtual game headset with goggles. He was treated conservatively for patella dislocation and had the OCF treated surgically. At 18 months follow up, his patella was stable and the OCF had healed.
\end{abstract}

Conclusion: OCF of posterolateral femur following patella dislocation is unusual. It must be suspected in patients who sustain patella dislocation whilst doing deep flexion maneuvers. Patella dislocation can conceal an unusual OCF.

Keywords: knee, femoral condyle, osteochondral, fracture, patellar dislocation
Volume I3 Issue 4 - 202 I

\section{Sefa W Canbilen, Khaldoun El-Abed, Riaz Ahmad}

Orthopaedics Department, University Hospitals Bristol and Weston NHS Foundation Trust

Correspondence: Khaldoun El-Abed FRCS, Orthopaedics Department, University Hospitals Bristol and Weston NHS Foundation Trust, Grange Road, Uphill, Weston Super Mare, United Kingdom, BS23 4TQ,

Email khaldoun.elabed@googlemail.com

\section{Introduction}

Lateral dislocation of the patella commonly occurs during early flexion, with contraction of Vastus Lateralis compressing the medial aspect of the patella onto the trochlear anterolateral articular surface generating a classical pattern of injury involving the medial patella and the non-weight-bearing area of the anterolateral femoral condyle. ${ }^{1}$ Patella dislocation associated with an OCF to the weight-bearing surface of the lateral femoral condyle is an even more unusual and rare lesion. ${ }^{1}$ OCF of the posterior surface of lateral femoral condyle has not been reported.

In this report, we present a case of Patella dislocation associated with an OCF of the posterior surface of lateral femoral condyle. This injury occurred during hyper-flexion of the knee whilst wearing a virtual reality (VR) headset with goggles. The patient was informed that data concerning the case would be submitted for publication, and he provided consent.

\section{Case report}

A healthy 37-year-old man sustained a knee injury after performing a deep flexion maneuver while playing a virtual reality game, with a VR headset on. His patella had dislocated laterally after he fell onto his knee whilst in deep flexion. There was no previous history of patella instability. On physical examination, the patient had a body mass index (BMI) of 33 and no signs of compartment syndrome. The patella was dislocated laterally, the knee was swollen, and tender over lateral distal femur. Radiographs of the left knee revealed a lipo-hemarthrosis and a large OCF of the lateral condyle of the femur (Figure $1 \& 2$ ). The patella was reduced in the emergency department and on repeat examination there was exquisite tenderness over proximal medial border of the patella in the area of the medial patella-femoral ligament (MPFL) attachment. Further tenderness was elicited over the lateral joint line. The patient was unable to straight leg raise and could hardly flex the knee to 30 degrees. The leg was placed in an extension splint and the patient was allowed non-weightbearing on crutches.

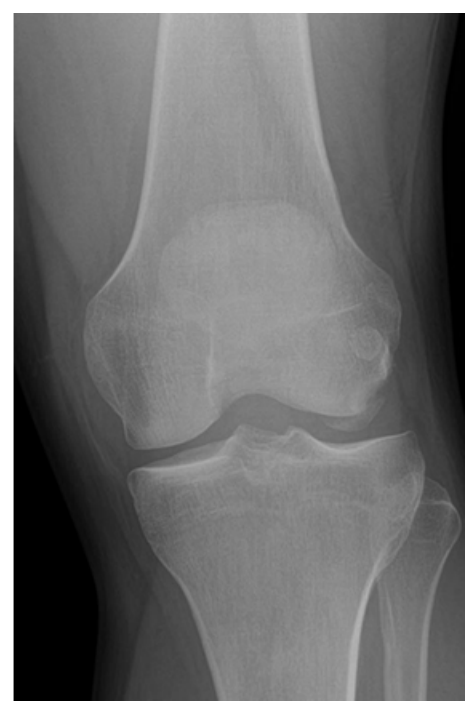

Figure I AP Radiograph Left Knee.

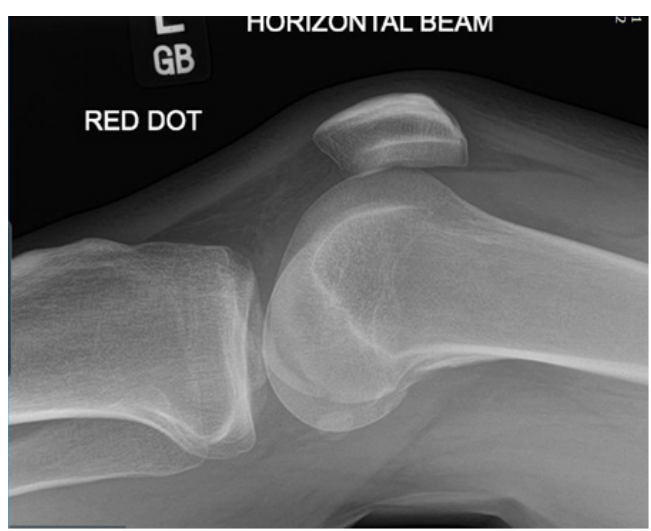

Figure 2 Lateral Radiograph Knee showing lateral femoral condylar osteochondral fracture. 
Computed tomography (CT) scan showed an OCF of the posterolateral femoral condyle (Figure $3 \& 4$ ). The MRI scan further confirmed the presence of an OCF of the lateral femoral condyle, and injury to the Medial Patellofemoral Ligament (MPFL), the latter is a secondary restraint to patella dislocation (Figure $5 \& 6$ ).

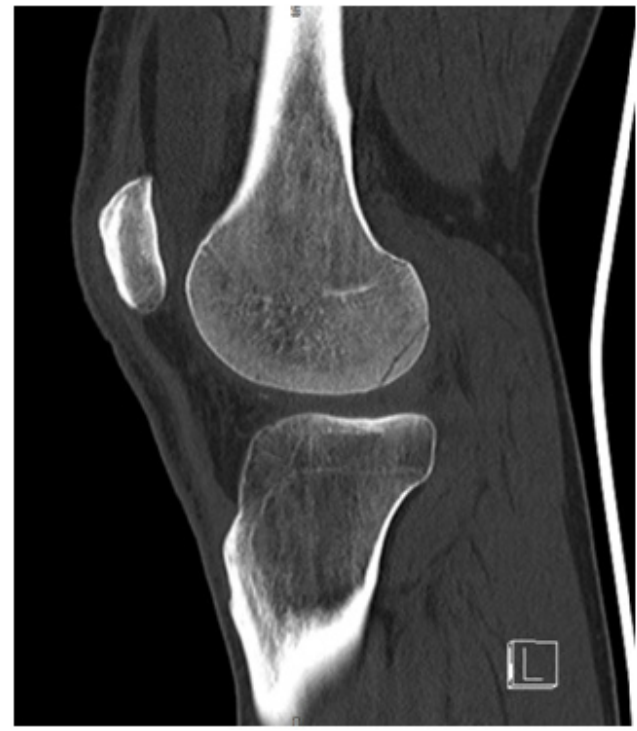

Figure 3 Sagittal CT Scan Left Knee.

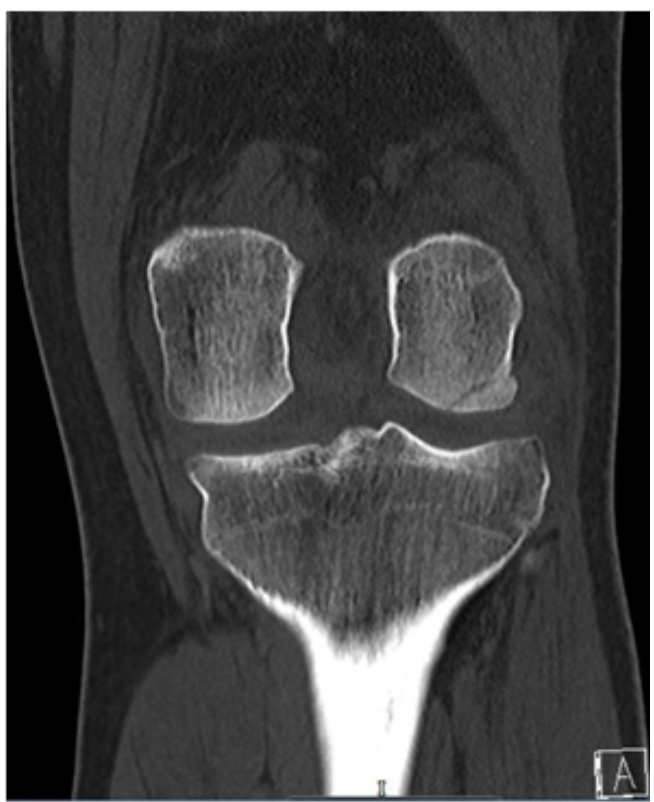

Figure 4 Coronal CT Scan view showing lateral femoral condylar osteochondral fracture.

Patella dislocation was treated conservatively. The patient was taken to operating room for fixation of the OCF. The OCF was hinged open and irrigated with saline (Figure 7). It was stabilized with $1.2 \mathrm{~mm}$ $\mathrm{K}$-wire. Fixation was achieved by using a $3 \times 22 \mathrm{~mm}$ Bio-compression screw (Arthrex) in the center followed by four $1.3 \times 18 \mathrm{~mm}$ chondral darts (Arthrex) around it (Figure 8).

Post-operatively the patient was allowed to mobilize non weight bearing with crutches on the affected side for 6 weeks. His knee was placed in a hinged knee brace locked in extension for the first 2 weeks followed by increase in range of motion (ROM) by 30 degrees at 2 weekly intervals. At 8 weeks post operatively, he was mobilized out of the brace and allowed weight bearing as tolerated and started physiotherapy rehabilitation program. At 6 months follow up, the knee was found to exhibit a full range of motion. The Patella was tracking centrally, and the patella apprehension test was negative. The radiographs confirmed healing of the OCF (Figure 9). The patient has not reported any issues since his surgery, 18 months ago.

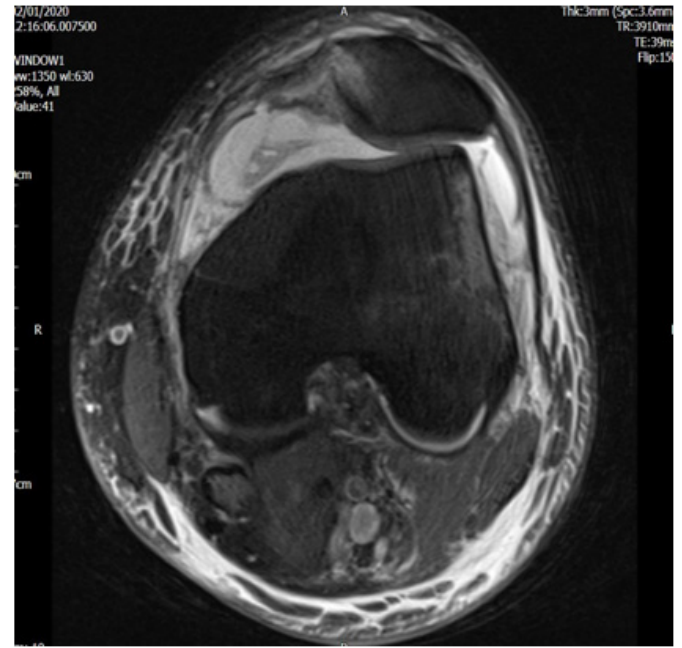

Figure 5 Axial MRI showing classical bruising pattern of patella dislocation \& MPFL injury.

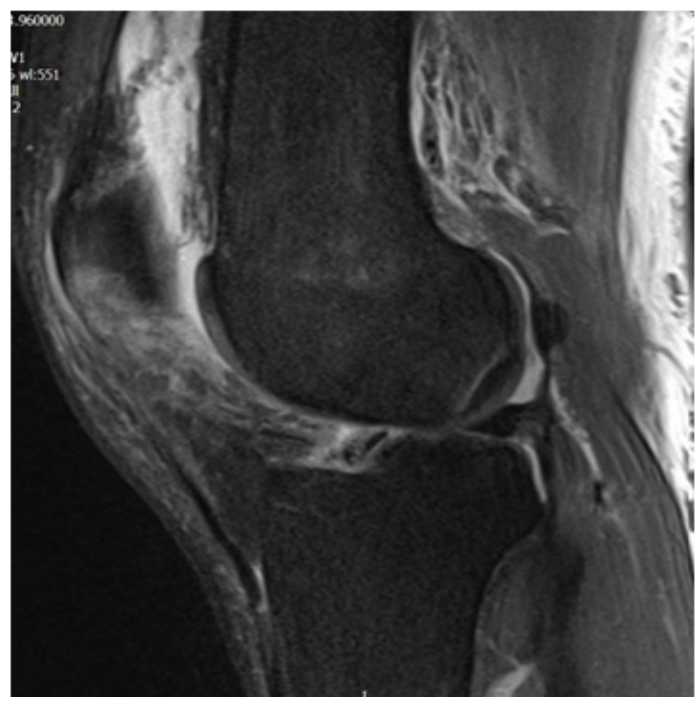

Figure 6 Sagittal MRI view showing lateral femoral condylar osteochondra fracture.

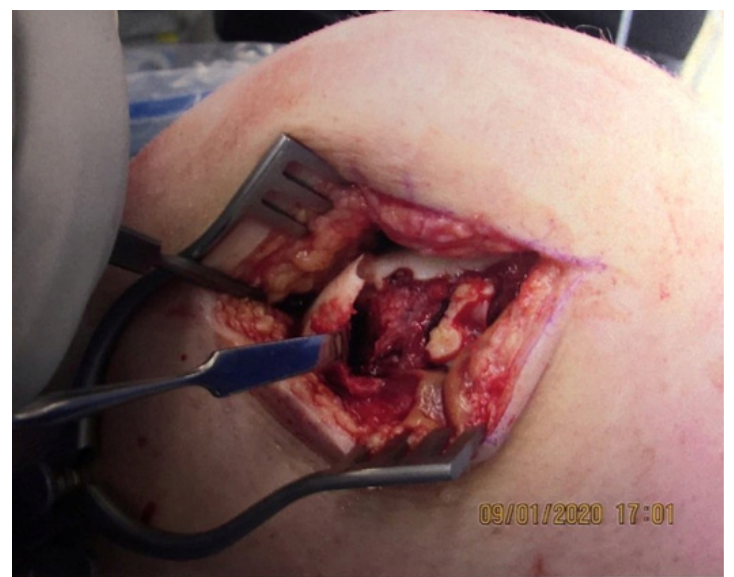

Figure 7 Intra-operative picture showing lateral femoral condylar osteochondral fracture. 


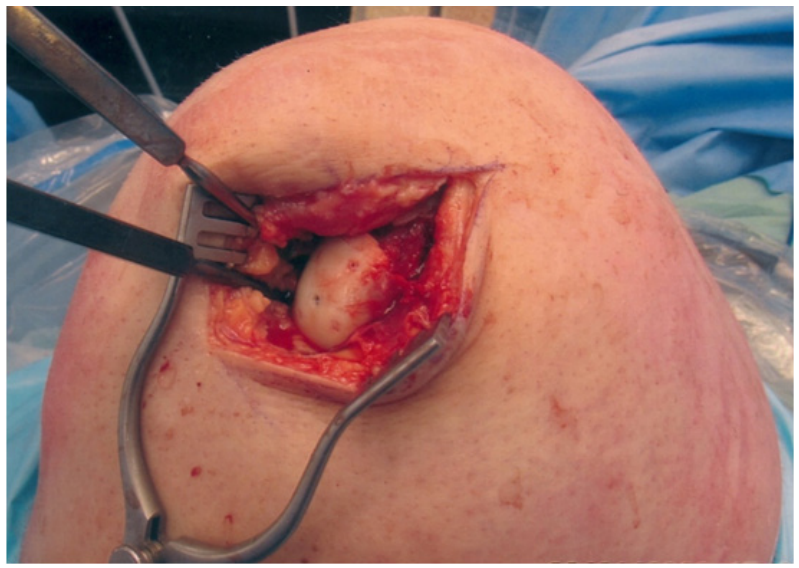

Figure 8 Intra-operative picture showing the lateral femoral condyle postfixation.

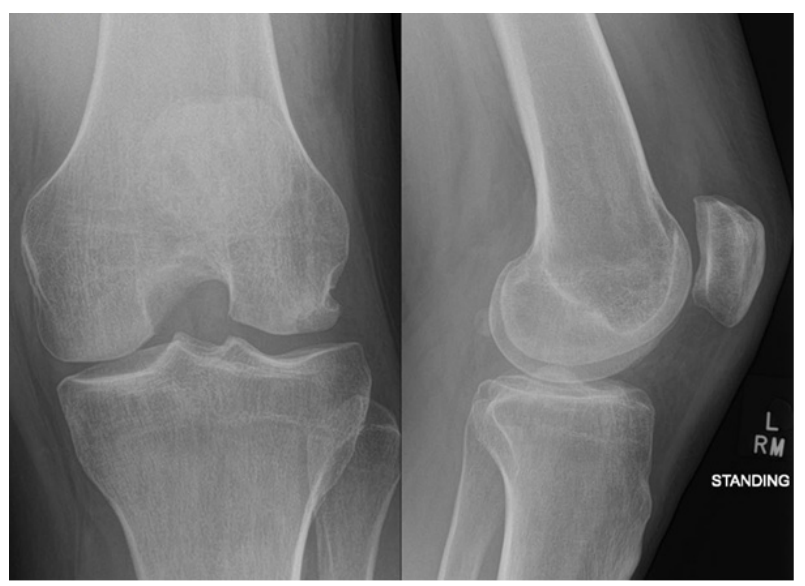

Figure 9 AP \& Lateral of Knee radiographs at I2-month follow up.

\section{Discussion}

Patellar dislocation has an average annual incidence of 5.8 per 100,000 in general population. ${ }^{2}$ The common mechanism of injury is forceful contraction of the quadriceps, with flexion and external rotation of the tibia on the femur. ${ }^{3}$ Less commonly, the patella can become dislocated from direct trauma with the knee in flexion. ${ }^{3}$ The patella is at risk of fracture both at the time of dislocation (OCF from the medial margin) and during relocation (OCF from the inferomedial facet). This accounts for a higher incidence of patella osteochondral injury. Osteochondral injury to the patella is a relatively common occurrence following lateral patellar dislocation of the knee. ${ }^{4}$ As the dislocated patella is reduced from a lateral to a medial direction by the pull of Vastus Medialis, the medial facet of the patella can impact the lateral femoral condyle which may result in fracture. ${ }^{5}$ This fracture is commonly at the anterosuperior surface of the lateral femoral condyle. Although large OCF may be seen on plain radiographs, smaller fragments may be undetected. Missed articular cartilage injury can lead to poor outcome.

Most of the published data refers to osteochondral injury of the medial patella facet as the main area that is involved. ${ }^{6-9}$ Some studies have also shown that an associated lateral femoral condyle osteochondral injury can be seen, but this is routinely on the far anterolateral portion of the lateral femoral condyle, often in the distal trochlear region. ${ }^{10-12}$

Various other authors have reported a $10 \%$ to $30 \%$ incidence of intra-articular osteochondral injuries seen on radiographs after patella dislocation, exclusive of patellar medial margin avulsion fracture ${ }^{1}$. In a consecutive series of 39 patient with acute patella dislocations, where the osteochondral damage was assessed both arthroscopically and macroscopically, the incidence of involvement of lateral femoral condyle was 12 patients $(31 \%)$. The commonest site involved was the anterosuperior surface of the femoral condyle ( 9 patients), followed by the lateral margin of the weight bearing zone ( 2 patients) and the lateral end of sulcus terminals (1 patient). ${ }^{1}$ In another study where the radiologic findings were correlated with arthroscopic findings the medial patella facet and anterosuperior surface of lateral femoral condyle were found to be the involved. ${ }^{13}$

Although not as common as osteochondral injuries to the medial facet of the patella and the anterior lateral portion of the lateral femoral condyle, osteochondral injury to the weight-bearing portion of the mid-lateral femoral condyle does occur with patella dislocation. A case series of 7 patients by Mashoof et al. described similar patellar dislocations with osteochondral fractures of the weight-bearing area on the lateral femoral condyle, produced by non-contact injuries. ${ }^{14}$ It was proposed that the injuries could result from patellar dislocation during deep flexion of the knee. The other proposed mechanism was shearing forces across the mid-lateral femoral condyle from tibiofemoral articulation coming in contact in valgus rotation. ${ }^{14}$

OCF in our case was in the unusual location of the posterolateral surface of the lateral femoral condyle resulting from patellar dislocation in a hyper-flexed knee. Furthermore, operative fixation as in this case is vital in prevention of an unfavorable outcome.

\section{Conclusion}

A posterolateral femoral OCF secondary to a patella dislocation, is a rare pathology, but must be evaluated in patients performing deep flexion maneuvers, with suspected patella dislocation. Spontaneously re-located patella dislocation in such cases can conceal unusual osteochondral injury.

\section{Acknowledgments}

None.

\section{Declarations}

\section{Consent for publication}

The patient has consented to the publication of this case report.

\section{Funding}

The authors received no funding for this project.

\section{Conflicts of interest}

The author states that there is no conflicts of interest.

\section{References}

1. Nomura E, Inoue M, Kurimura M. Chondral and osteochondral injuries associated with acute patellar dislocation. Arthroscopy. 2003;19(7):717721.

2. Fithian DC, Paxton EW, Stone ML, et al. Epidemiology and natural history of acute patellar dislocation. Am J Sports Med. 2004;32(5):1114-1121.

3. Abramov M, Stock H. Lateral patellar dislocation: mechanism of disease, radiographic presentation, and management. Conn Med. 2013;77(4):235238.

4. Lee BJ, Christino MA, Daniels AH, et al. Adolescent patellar osteochondral fracture following patellar dislocation. Knee Surg Sports Traumatol Arthrosc. 2013;21(8):1856-1861. 
5. Seeley MA, Knesek M, Vanderhave KL. Osteochondral injury afte acute patellar dislocation in children and adolescents. $J$ Pediatr Orthop. 2013;33(5):511-518.

6. Bassett FH. Acute dislocation of the patella, osteochondral fractures, and injuries to the extensor mechanism of the knee. Instr Course Lect. $1976 ; 25: 40-49$

7. Cash JD, Hughston JC. Treatment of acute patellar dislocation. Am J Sports Med. 1988;16(3):244-249.

8. Cofield RH, Bryan RS. Acute dislocation of the patella: results of conservative treatment. J Trauma. 1977;17(7):526-531.

9. Hawkins RJ, Bell RH, Anisette G. Acute patellar dislocations. The natural history. Am J Sports Med. 1986;14(2):117-120.
10. Faber KJ, Dill JR, Amendola A, et al. Occult osteochondral lesions after anterior cruciate ligament rupture. Six-year magnetic resonance imaging follow-up study. Am J Sports Med. 1999;27(4):489-494.

11. Makin M. Osteochondral fracture of the lateral femoral condyle. J Bone Joint Surg Am. 1951;33 A(1):262-264.

12. Rosenberg NJ. Osteochondral fractures of the lateral femoral condyle. $J$ Bone Joint Surg Am. 1964;46:1013-1026.

13. Stanitski CL, Paletta GA Jr. Articular cartilage injury with acute patellar dislocation in adolescents. Arthroscopic and radiographic correlation. Am J Sports Med. 1998;26(1):52-55.

14. Mashoof AA, Scholl MD, Lahav A, et al. Osteochondral injury to the midlateral weight-bearing portion of the lateral femoral condyle associated with patella dislocation. Arthroscopy. 2005;21(2):228-232. 